\title{
Effekt von Ambroxol auf die Surfactantsekretion und -synthese von isolierten, alveolären Typ-II-Zellen
}

\begin{abstract}
Zusammenfassung: Ein Teil der Wirkung von Ambroxol wird auf eine Stimulation des Surfactantsystems zurückgeführt. Die Untersuchungen, die dem zugrunde liegen, geben eher indirekte Hinweise auf Surfactantsynthese und -sekretion. Ein direkter Nachweis der Stimulation von alveolären Typ II Zellen im Hinblick auf Synthese und Sekretion fehlt. In dieser Untersuchung wurde eine direkter Effekt von Ambroxol auf die Surfactantsynthese und -sekretion untersucht. Dazu wurde die Freisetzung von markiertem Phosphatidylcholin unter dem Einfluss von Ambroxol in ansteigender Dosierung $\left(10^{-8} \mathrm{M}\right.$ bis $\left.10^{-4} \mathrm{M}\right)$ und mit zunehmender Expositionszeit gemessen. Es fand sich weder mit zunehmender Konzentration noch mit zunehmender Zeit ein signifikanter Effekt des Ambroxol auf die Surfactantsekretion. Als Marker der Surfactantsynthese wurde die Aufnahme von ${ }^{3} \mathrm{H}$-markiertem Cholin und der Einbau in ${ }^{3} \mathrm{H}$-Phosphatidylcholin (PC) untersucht. Hier wurden ebenfalls ansteigende Konzentrationen $\left(10^{-7} \mathrm{M}\right.$ bis $\left.10^{-5} \mathrm{M}\right)$ untersucht, und der zelluläre Gehalt an PC über 1, 2, 4, 8, 24 h verfolgt. Zu keinem Zeitpunkt fand sich eine signifikante Stimulation dieses Parameters durch Ambroxol in den getesteten Konzentrationen. Höhere Konzentrationen als die hier getesteten führten zu vermehrter LDH Freisetzung aus den isolierten AT-II-Zellen. Ambroxol, das auch antioxidative und antiinflammatorische Wirkungen besitzt, scheint also keine direkte Wirkung auf isolierte AT-II-Zellen (der Ratte) zu besitzen, wie dies zum Beispiel für die $\beta_{2}$-Mimetika zutrifft. Eine spezifische, pharmakologische Möglichkeit der Stimulation des Surfactantsystems gilt es weiterhin zu entwickeln.
\end{abstract}

Effect of ambroxol on surfactant synthesis and secretion in isolated type II alveolar cells: The effect of ambroxol is attributed in part to an effect on surfactant synthesis and secretion. Evidence supporting this hypothesis is largely indirect; a direct effect of ambroxol on surfactant synthesis and secretion remains to be demonstrated. In this study a direct effect of ambroxol was evaluated using isolated alveolar type II cells. Secretion of labelled phosphatidylcholine was measured following the addition of increasing concentrations $\left(10^{-8} \mathrm{M}\right.$ to $\left.10^{-4} \mathrm{M}\right)$ of ambroxol to the culture medium for increasing time intervals. There was no significant increase in surfactant secretion with increased ambroxol concentration or prolonged exposure time. Uptake of ${ }^{3} \mathrm{H}$-choline and synthesis into ${ }^{3} \mathrm{H}$-phsophatidylcholine was analyzed as an indicator of surfactant synthesis. Again, in-

Pneumologie 2000; 54: 278-283

(c) Georg Thieme Verlag Stuttgart · New York ISSN 0934-8387

\section{H. R. Wirtz}

Medizinische Klinik I und Poliklinik, Universität Leipzig creasing concentrations of ambroxol $\left(10^{-7} \mathrm{M}\right.$ to $\left.10^{-5} \mathrm{M}\right)$ were followed for 1, 2, 4, 8 and $24 \mathrm{~h}$. There was no significant effect on synthesis at any time point. Concentrations higher than those mentioned here resulted in LDH release from cultured cells. Ambroxol which also has anti-oxidative and anti-inflammatory effects does not exhibit direct stimulatory effects on surfactant synthesis and secretion in isolated (rat) alveolar type II cells as has been demonstrated e.g. for $\beta_{2}$-adrenergic stimulation. $A$ specific pharmacologic way to stimulate the surfactant system remains to be developed.

Das im Jahre 1979 eingeführte Medikament Ambroxol wird in der Inneren Medizin, besonders der Pneumologie, aber auch der Pädiatrie, der Chirurgie und in der Intensivmedizin häufig eingesetzt. Die Indikation ist zumeist die Vermeidung pulmonaler Komplikationen wie lokale Atelektasenbildung z.B. bei der maschinellen Beatmung, bei perioperativen Ventilationsstörungen, aber auch die sekretolytische Therapie bei der chronischen Bronchitis, ja sogar das Asthma bronchiale findet sich in der Sparte „Anwendungsgebiete“ der derzeit gültigen Roten Liste (Rote Liste ${ }^{\circledR}$ 2000, Editio Cantor Verlag, Aulendorf). Neben antiinflammatorischen und antioxidativen Eigenschaften soll Ambroxol diese Wirkungen vor allem durch seinen Effekt auf das Surfactantsystem der Lunge besitzen, - insbesondere die Synthese und die Sekretion des Surfactant stimulieren. Effekte auf das Surfactantsystem der Lunge wurden zumeist im Tierversuch und im Modell der isolierten Lunge, häufig anhand von morphometrischen Analysen untersucht. Ergebnisse von Tierversuchen unterstützen die Aussage, dass Ambroxol die Surfactantsynthese und/oder -sekretion stimuliert. Zu der Zeit, da diese Untersuchungen durchgeführt wurden, waren Versuche mit isolierten AT-II-Zellen noch nicht in nennenswertem Umfang verfügbar. In zwei frühen Untersuchungen mit isolierten AT-II-Zellen wurde unter Einwirkung von Ambroxol ein vermehrter Einbau von markierten Vorläufersubstanzen in Phospholipide gezeigt $[1,2]$. Dies deutet in Übereinstimmung mit dem zuvor Genannten auf eine Stimulation der Surfactantsynthese.

Inzwischen sind Versuche mit isolierten AT-II-Zellen zu einem Standard für die Untersuchung der Fähigkeit zur Stimulation der Surfactantsekretion und -synthese geworden $[3,4]$. In dieser Untersuchung wurden daher die direkten Effekte von Ambroxol auf die Surfactantsynthese und -sekretion von isolierten alveolären Typ-II-Zellen in primärer Kultur untersucht. 


\section{Material und Methoden}

Dulbeccos modifiziertes Eagle Medium (DMEM) stammte von Gibco (Eggenstein). L-Glutamin, Penicillin, Gentamicin, Immunoglobulin G (Ratte) (IgG), Desoxyribonuclease (DNase) I, Trypsin, Ethylenglycol-tetraessigsäure (EGTA), Adenosintriphosphate (ATP), Terbutalin und 12-O-Tetradecanoylphorbol13-azetat (TPA) wurden von Sigma (Deisenhofen), ${ }^{3} \mathrm{H}$-markiertes Cholin von Amersham (Amersham Pharmacia Biotech Europe $\mathrm{GmbH}$, Freiburg) bezogen. Elastase (vom Schweinepankreas) kauften wir von Elastin Products Co. (EPC, Owensville, USA). Ambroxol Reinsubstanz (MW 414.6; Mucosol$\operatorname{van}^{\mathrm{TM}}$ ) wurde freundlicherweise von Boehringer Ingelheim (Ingelheim) zur Verfügung gestellt. Wir setzten in den Versuchen sowohl die pulverförmige Reinsubstanz als auch die kommerziell erhältliche, fertige Infusionslösung ein. Da die Ergebnisse mit der Infusionslösung praktisch mit denen der Reinsubstanz übereinstimmten, werden sie hier nicht dargestellt.

\section{Zellkultur}

Männliche Sprague-Dawley-Ratten wurden mit einem Gewicht von 150-200 g bestellt. Nach Entnahme der Lungen wurden AT-II-Zellen nach der von Dobbs et al. [5] beschriebenen Methode unter Verwendung des Enzyms Elastase und durch differenzielle Adhäsion auf Ratten-IgG-beschichteten Kulturschalen isoliert. Eigene Ergebnisse mit dieser Technik der Zellisolierung wurden veröffentlicht [z.B. 6].

7,5 $\times 10^{5}$ AT-II-Zellen pro $35 \mathrm{~mm}$ Kulturschale wurden dabei in DMEM mit $10 \%$ fötalem Kälberserum (FCS), Penicillin (100 U/ $\mathrm{ml})$, Gentamicin $(50 \mu \mathrm{g} / \mathrm{ml})$ und L-Glutamin $(2 \mathrm{mM})$ über $22 \mathrm{~h}$ im Inkubator bei $37^{\circ} \mathrm{C}, 10 \% \mathrm{CO}_{2}$ in Raumluft inkubiert. In dieser Zeit hatten die Zellen Gelegenheit zur Adhäsion. Im Falle einer Ambroxol Präinkubation wurde Ambroxol Reinsubstanz $\left(10^{-8} \mathrm{M}, 10^{-7} \mathrm{M}, 10^{-6} \mathrm{M}, 10^{-5} \mathrm{M}\right.$ und $\left.10^{-4} \mathrm{M}\right)$ in DMSO/ $\mathrm{NaCl}$ (maximale DMSO Konzentration im Zellkulturmedium 0,05\%) bzw. kommerziell erhältliche Ambroxol-Fertiglösung in den gleichen Konzentrationen entweder $4 \mathrm{~h}$ oder $24 \mathrm{~h}$ vor dem Sekretionsexperiment dem Kulturmedium zugegeben. Kontrollen erhielten in diesem Zeitraum eine äquivalente Menge DMSO (vergleichbar der höchsten Konz. Ambroxol, also auch der höchsten Menge DMSO).

Für Sekretionsexperimente wurde dem Medium während der 22-h-Inkubation bis zur Adhärenz noch $1 \mu \mathrm{Ci}{ }^{3} \mathrm{H}$-Cholin pro $\mathrm{ml}$ Kulturmedium (1 $\mathrm{ml}$ Kulturmedium/35 mm Kulturschale) zugegeben. Im Fall der Bestimmung der Surfactantsynthese durch Aufnahme eines markierten Substrates wurde ${ }^{3} \mathrm{H}-$ Cholin dem Medium erst nach der Adhärenz für die unten aufgeführte Zeitdauer beigefügt.

\section{Messung der Surfactantsekretion}

Im Anschluss an die Adhärenz und Markierung wurden die Zellen mit frischem DME Medium gewaschen und eine exakte Menge Medium appliziert. Kurz darauf wurden die Agonisten aus 80fach konzentrierten Stammlösungen zugegeben $\left(10^{-4} \mathrm{M}\right.$ Terbutalin, $10^{-8} \mathrm{M}$ TPA und $10^{-4} \mathrm{M}$ ATP; jeweils Endkonzentration im Kulturmedium; zusammen: „TTA“). Kontrollen erhielten $\mathrm{NaCl}$. Während der eigentlichen Sekretionsphase enthielt das Medium dieselbe Konzentration Ambroxol wie während der Präinkubation außer in den Experimenten, in denen die akute Wirkung von Ambroxol und damit ausschließlich während der Sekretionsperiode untersucht wurde. In allen Fällen betrug die Sekretionsperiode $2 \mathrm{~h}$. Anschließend wurden Medium und Zellen getrennt geerntet.

${ }^{3} \mathrm{H}$-markiertes Phosphatidylcholin (PC) als Markersubstanz des Surfactant wurde sowohl im Überstand als auch in der zellulären Fraktion nach mechanischer Entfernung der Zellen unter Ethanol bestimmt. Dazu wurde zunächst jeweils eine Lipidextraktion durchgeführt [7] und anschließend die Radioaktivität im Überstand und in der zellulären Fraktion mittels Szintillation gemessen. Die Sekretion wurde errechnet indem die Aktivität (Counts) im Überstand als prozentualer Anteil der Gesamtaktivität (Überstand und zelluläre Fraktion) ausgedrückt wurde. Somit wird die Sekretion sowohl basal als auch unter Stimulation durch TTA prozentual ausgedrückt (d.h. der freigesetzte Anteil der insgesamt vorhandenen, lipidextrahierbaren Tritium-Aktivität).

\section{Bestimmung der Surfactantsynthese}

In Umkehrung zum Vorgehen bei der Sekretion wurde hierbei die Inkorporation von ${ }^{3} \mathrm{H}$-Cholin in ${ }^{3} \mathrm{H}$-Phosphatidylcholin $\left({ }^{3} \mathrm{H}-\mathrm{PC}\right)$ als Marker der Surfactantsynthese bestimmt. Dazu wurden Zellen im Anschluss an die Adhärenzzeit mit $1 \mu \mathrm{Ci}$ ${ }^{3} \mathrm{H}-\mathrm{Cholin} / \mathrm{ml}$ Medium und Kulturschale für den angegebenen Zeitraum inkubiert (24-48 h nach Isolation). Nach Beendigung dieser Syntheseperiode wurden die Zellen sorgfältig mit Medium gewaschen und die Aktivität in der zellulären Fraktion nach mechanischer Ernte unter Ethanol und einer Lipidextraktion wie oben durch Szintillationsbestimmung gemessen. Die Aktivität in der zellulären Fraktion wurde als prozentualer Anteil der dem Medium ursprünglich zugegebenen Aktivität ausgedrückt.

\section{Bestimmung der Lactatdehydrogenaseaktivität}

Die Aktivität der Lactatdehydrogenase (LDH) als Marker für eine Schädigung der Zellen wurde im Überstand aller in diesen Experimenten ausgewerteten Kulturschalen durchgeführt und verglichen zu der Aktivität der zellulären Fraktion von drei nicht radioaktiv markierten Referenzkulturschalen, die völlig identisch behandelt, in jedem Experiment mitgeführt wurden. Die LDH Aktivität im Überstand und damit der freigesetzte Anteil wurde als prozentualer Anteil der gesamten LDH Aktivität (Überstand und zelluläre Fraktion) ausgedrückt. LDH wurde fluorimetrisch gemessen [8]. Eine Freisetzung von mehr als $2 \%$ wurde als Indikator für eine nicht akzeptable Zellschädigung angenommen.

\section{Ergebnisse}

\section{Surfactantsekretion}

Die basale ${ }^{3} \mathrm{H}-\mathrm{PC}$ Sekretion in unstimulierten AT-II-Zellkulturen ohne Vorinkubation oder Zugabe von Ambroxol während der Sekretionsperiode betrug $1,79 \% \pm 1,03(n=7$ Zellisolierungen, Mittel \pm SD).

Der gemeinsame Effekt von Terbutalin, Adenosintriphosphat und 12-0-Tetradecanoylphorbol-13-Azetat (TTA), die alle über unterschiedliche Signalwege auf komplimentäre Weise die 

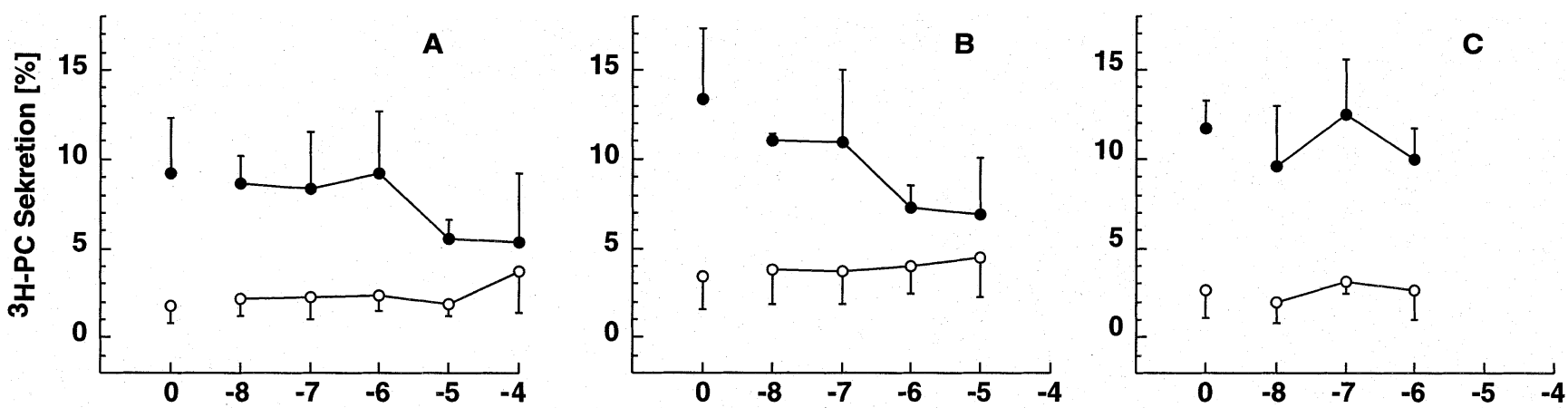

Ambroxol [10X $\mathrm{M}]$

Abb. 1 PC Sekretion von unstimulierten (offene Punkte) und mit ATT stimulierten (geschlossene Punkte) AT II Zellen ohne („0“) und mit ansteigenden Konzentrationen von Ambroxol im Kulturmedium (Mittelwerte \pm SD): Ohne Vorinkubation: A, 4h Vorinkubation: B; $24 \mathrm{~h}$ Vorinkubation: $C$.

Sekretion stimulieren und daher gemeinsam als maximaler pharmakologischer Sekretionsreiz gelten können, ist in Abb.1 dargestellt. Die basale Sekretion ließ sich in dieser Serie von Experimenten im Mittel um das 5,2fache steigern (abs.: $1,79 \% \pm 1,03$ auf $9,25 \% \pm 3,1 ; n=7, p<0,05)$.

Die Zugabe von Ambroxol Reinsubstanz in Konzentrationen zwischen $10^{-8} \mathrm{M}$ und $10^{-3} \mathrm{M}$ zum Kulturmedium direkt vor der zweistündigen Sekretionsphase (keine Vorinkubation) führte nicht zu einer signifikanten Steigerung der basalen oder der stimulierten Surfactantsekretion (Abb.1A). In einer Konzentration von $10^{-4} \mathrm{M}$ und $10^{-3} \mathrm{M}$ kam es zu einer deutlich gesteigerten Freisetzung von LDH, was bei Fehlen anderer Faktoren als ein direkt zellschädigender Effekt von Ambroxol in diesem in vitro Modell gewertet wurde.

\section{Effekt einer Vorinkubation mit Ambroxol} auf die Surfactantsekretion

Der Effekt einer 4-stündigen und einer 24-stündigen Vorinkubation mit ansteigenden Konzentrationen von Ambroxol (Reinsubstanz und Mucosolvan Infusionslösung ${ }^{\circledR}$ ) ist in den Abbildungen 1B und 1C dargestellt. In beiden Fällen wurde ebenfalls keine signifikante Veränderung der Surfactantsekretion beobachtet. Nach einer Vorinkubation von $4 \mathrm{~h}$ wurde allerdings ein Trend $\mathrm{zu}$ einer verminderten stimulierten Surfactantsekretion nach Zugabe von $10^{-6}$ und $10^{-5} \mathrm{M}$ Ambroxol im Vergleich zur stimulierten Sekretion ohne Ambroxol beobachtet (Kruskal-Wallis-Test, $n=3$ Zellisolierungen, $\mathrm{p}=0,09$ ). Zudem war eine deutlichere LDH-Freisetzung bei $10^{-4} \mathrm{M}$ Ambroxol zu beobachten und nach $24 \mathrm{~h}$ Präinkubation schon eine vermehrte LDH-Freisetzung bei $10^{-5} \mathrm{M}$ Ambroxol, so dass diese Konzentrationen nach Präinkubation mit Ambroxol nicht mehr auszuwerten waren.

\section{Surfactantsynthese}

Die Aufnahme von extern zugegebenem tritiummarkierten Cholin $\left({ }^{3} \mathrm{H}-\mathrm{C}\right.$, hydrophil) und der Einbau in das Surfactantphospholipid-Phosphatidylcholin ( ${ }^{3} \mathrm{H}-\mathrm{PC}$, lipophil) wurden als Marker für die Surfactantsynthese bestimmt.
Die Aufnahme von ${ }^{3} \mathrm{H}-\mathrm{C}$ war in unstimulierten, nicht mit Ambroxol behandelten Zellen während der ersten acht Stunden schneller als in dem darauf folgenden Beobachtungszeitraum von $16 \mathrm{~h}$ ( $1 \mathrm{~h}: 0,5 \% \pm 0,1 ; 2 \mathrm{~h}: 1,5 \% \pm 0,5 ; 4 \mathrm{~h}: 3,1 \% \pm 0,6$; 8 h: 7,2\% $\pm 2,9 ; 24$ h: 15,3\% $\pm 5,0$; Abb. 2). Der Ambroxoleffekt wurde in den Konzentrationen $10^{-7} \mathrm{M}$ bis $10^{-5} \mathrm{M}$ untersucht. Noch höhere Konzentrationen wurden aufgrund der Erfahrungen im Hinblick auf die Zellschädigungen in den Sekretionsexperimenten nicht eingesetzt. Sowohl ohne als auch mit Ambroxol war die ${ }^{3} \mathrm{H}-\mathrm{C}$ Aufnahme nach spätestens $8 \mathrm{~h}$ gegenüber der Situation nach einer Stunde signifikant gesteigert. Im Vergleich mit der den unstimulierten, nicht mit Ambroxol behandelten Zellen kam es jedoch bei keiner der eingesetzten Konzentrationen von Ambroxol zu irgendeinem Zeitpunkt zu einer signifikanten $\mathrm{Zu}$ - oder Abnahme

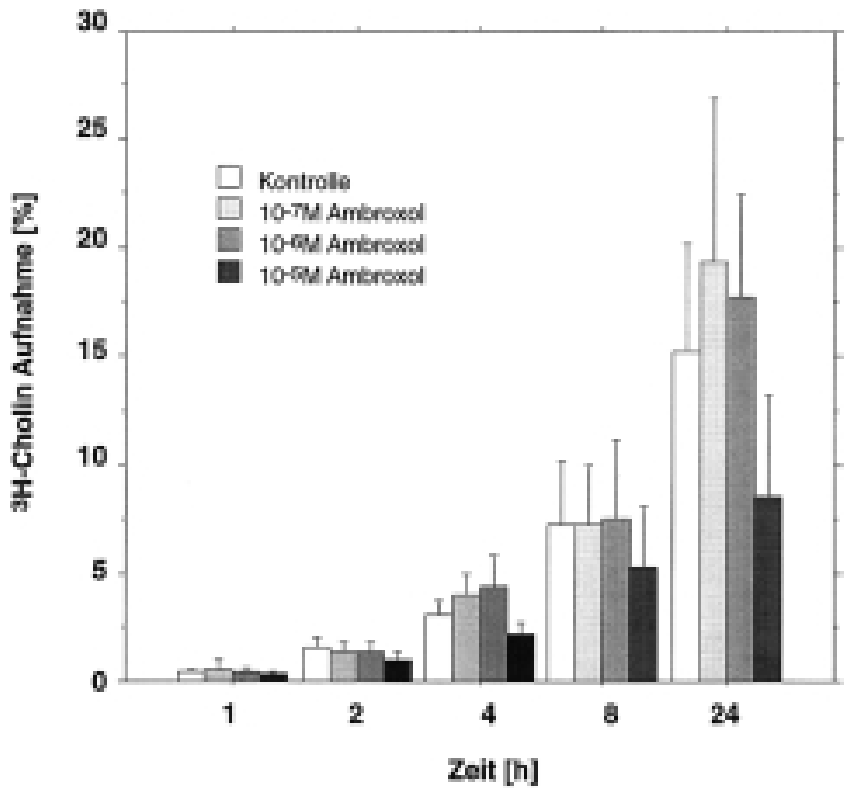

Abb. 2 Einbau von ${ }^{3} \mathrm{H}$-markiertem Cholin in Phosphatidylcholin mit ansteigender Inkubationszeit in Abwesenheit (Kontrolle) bzw. in Gegenwart von ansteigenden Konzentrationen von Ambroxol während des dargestellten Zeitraumes (Mittelwerte \pm SD). 
der als ${ }^{3} \mathrm{H}-\mathrm{PC}$ gemessenen Radioaktivität. Die Gruppenvergleiche (Vergleich der Ambroxolkonzentrationen zu jedem Zeitpunkt) wurden mit dem Kruskal-Wallis-Test durchgeführt. Dieser zeigte lediglich in einem Fall einen signifikanten Unterschied an: Nach $4 \mathrm{~h}$ fand sich ein signifikanter Unterschied zwischen Zellen, die mit $10^{-7} \mathrm{M}$ bzw. $10^{-6} \mathrm{M}$ Ambroxol und solchen, die mit $10^{-5} \mathrm{M}$ behandelt worden waren. $\mathrm{Zu}$ den nicht behandelten Kontrollen bestand jedoch kein signifikanter Unterschied. Zusammenfassend zeigte sich eine statistisch nicht signifikante Tendenz zur geringen Erhöhung des ${ }^{3} \mathrm{H}-\mathrm{PC}$ Einbaus bei den niedrigeren Konzentrationen von Ambroxol $\left(10^{-7} \mathrm{M}\right.$ bzw. $\left.10^{-6} \mathrm{M}\right)$, und eine etwas deutlichere Tendenz zur Verminderung des ${ }^{3} \mathrm{H}-\mathrm{PC}$ Einbaus bei den höheren Konzentrationen von Ambroxol $\left(10^{-5} \mathrm{M}\right)$.

\section{Diskussion}

In dieser Untersuchung zu den Effekten von Ambroxol auf die Sekretion und Synthese des Surfactant in einem AT II Zellkulturmodell konnte kein signifikanter Effekt von Ambroxol auf die Sekretion oder die Synthese des pulmonalen Surfactant nachgewiesen werden. Dabei wurden sowohl die basale Sekretion als auch die stimulierte Sekretion untersucht. Die Bedeutung der basalen Sekretion im Zellkulturmodell für die Situation in vivo ist unklar. Dagegen reflektiert die sekretorische Antwort auf Agonisten vermutlich auch die Stimulierbarkeit in vivo [4]. Eine Übertragung der Ergebnisse eines Invitro-Modells auf die Situation in vivo ist meist problematisch. Neben dem Fehlen organspezifischer und systemischer Einflüsse spielt auch der Speziesunterschied (Rattenzellen versus humane Lunge) eine bislang nicht gut charakterisierte Rolle.

In den hier dargestellten Versuchen wurde eine gleichzeitige Stimulierung über unterschiedliche Signalwege gewählt (Terbutalin: cAMP; TPA: Diacylglyzerol, ATP: cAMP, $\mathrm{IP}_{3}$ und weiter $\mathrm{Ca}^{++}$, Diacylglyzerol). Damit wird in diesem Modell der isolierten AT II Zellen meiner Erfahrung nach eine maximale Stimulierung erreicht. Eine solche maximale Steigerung der Sekretion ist dann notwendig, wenn auch ein die Sekretion vermindernder Einfluss als Reaktion in Frage kommt, wie es zum Beispiel für den Zigarettenrauch im gleichen Modell gezeigt werden konnte [6]. Dagegen wäre ein auch nur gering sekretionssteigernder Einfluss insbesondere bei der basalen Sekretion deutlich geworden. Der fehlende Einfluss von Ambroxol auf beide Situationen steht im Widerspruch zu älteren Daten, die eine gesteigerte Surfactantsekretion durch Ambroxol nahe legen. Viele der älteren Arbeiten, auf die sich diese Aussagen gründen, bieten jedoch nur indirekte Hinweise auf einen sekretionsfördernden Effekt von Ambroxol. So wurde bei Wistar Ratten nach Gabe von Ambroxol in einer Dosis von 100 und $200 \mathrm{mg} /$ die über 3 und 6 Tage ein Anstieg im Verhältnis des Volumenanteils der AT-II-Zellen zum restlichen alveolären Gewebe beobachtet. Dabei kam es jedoch nur zu einem geringen Anstieg des Anteils der Lamellarkörperchen, so dass richtigerweise von den Autoren bemerkt wurde, dass ein relativer Zuwachs des AT-II-Zellanteils nicht notwendigerweise ein Indiz für eine vermehrte Surfactantproduktion sei [9]. AT-II-Zellen reagieren auf eine Vielzahl von schädigenden Einflüssen im Alveolarbereich mit einer Hypertrophie und Hyperplasie, so zum Beispiel bei fibrosierenden Lungenerkrankungen aber eben auch beim akuten Lungenschaden [10]. Andere Arbeiten beschreiben mit morphologi- schen Methoden einen Schutz vor dem schädigenden Einfluss von Bleomycin durch Ambroxol und eine beschleunigte Reifung der AT-II-Zellen in fetalen Rattenlungen (siehe Übersicht über morphologische Arbeiten in: [11]). Solche Effekte des Ambroxol lassen sich mit der heute bekannten antioxidativen und antiinflammatorischen Wirkung des Ambroxols besser begründen, als mit einer Wirkung auf das Surfactantsystem $[12,13,14,15,16,17,18,19,20]$.

In einem Modell der perfundierten Rattenlunge wurde eine vermehrte Inkorporation von ${ }^{14} \mathrm{C}$-Cholin und ${ }^{3} \mathrm{H}$-Glycerol in Phosphatidylcholin (PC), doppelt gesättigtes Phosphatidylcholin and Phosphatidylglycerol beobachtet, nachdem die Tiere zuvor einen, bzw. drei Tage lang $12,5 \mathrm{mg} / \mathrm{kg}$ KG Ambroxol erhalten hatten [21]. Die Inkorporation in die Surfactantassoziierten Phospholipide nach dreitägiger Gabe war 2,5fach bzw. 2,1fach und 2fach gesteigert, während die Steigerung der Inkorporation in die Lunge insgesamt 1,7fach gesteigert war. In einer anderen Untersuchung wurde eine vermehrte radioaktive Markierung von Lamellarkörperchen in erwachsenen Ratten beschrieben, die mit $200 \mathrm{mg} / \mathrm{kg} \mathrm{KG}$ Ambroxol vorbehandelt waren [22]. Aus diesen Daten lassen sich allenfalls Hinweise auf eine möglicherweise gesteigerte Surfactantsynthese entnehmen, zumal jeweils der Abbauweg des Surfactant, sowie die Sekretion nicht untersucht wurden.

Hinweise auf eine vermehrte Freisetzung von Surfactant nach Gabe von Ambroxol gaben zum Beispiel frühe Versuche an Meerschweinchen, deren Lungen in Abständen mehrfach gewaschen wurden [23], und die eine Stunde vor der ersten Lavage $20 \mu \mathrm{Ci} \mathrm{C}^{14}$-markiertes Cholin intraperitoneal erhielten. Es fand sich dabei eine deutliche Steigerung der Radioaktivität in allen, einschließlich der ersten Lavage der mit Ambroxol vorbehandelten Tiere im Vergleich zu den Kontrollen. In anderen Untersuchungen fand sich analog eine vermehrte spezifische Aktivität von unterschiedlichen Phospholipidspezies, meist unter Einschluss des wesentlichen Surfactantbestandteils, des doppelt gesättigten Phosphatidylcholins [21, 24, 25].

Nur in einer anderen Untersuchung wurden AT-II-Zellen von erwachsenen, nicht fetalen Rattenzellen untersucht: Diese wurden über mindestens 4 Tage mit Ambroxol vorinkubiert und damit doppelt so lange wie in den hier vorgestellten Experimenten kultiviert, über $48 \mathrm{~h}$ mit ansteigenden Dosen $(1-16 \mu \mathrm{g} / \mathrm{ml})$ Ambroxol inkubiert und erhielten ${ }^{14} \mathrm{C}$-Azetat über diesen Zeitraum. Anschließend wurde vor Anbruch der letzten Stunde der Inkubation noch ${ }^{3} \mathrm{H}$-Azetat zur Bestimmung des Anteils des Umsatzes (i.e. ${ }^{3} \mathrm{H}$-Inkorp/ $/{ }^{14} \mathrm{C}$-Inkorp.*100) zugegeben. Es fand sich dabei eine dosisabhängige, ab $4 \mu \mathrm{g} / \mathrm{ml}$ beginnende und über $8 \mu \mathrm{g} / \mathrm{ml}$ wieder abnehmende Steigerung der ${ }^{14} \mathrm{C}$-Inkorporation in diesen Kulturen, ein abnehmender Umsatzanteil unter steigender Ambroxol-Dosierung für Phosphatidylglycerol aber tendenziell auch für die anderen surfactantspezifischen Phospholipide [2]. Es ist also denkbar, dass zumindest ein Teil der mehrfach beobachteten, vermehrten Inkorporation von markierten Surfactant-Vorläufermolekülen in Surfactantphospholipide unter Ambroxol durch einen verminderten Abbau von bereits vorhandenem Surfactant und dadurch einen verminderten Einbau wiederverwendeter und daher noch nicht markierter Surfactantbestandteile bedingt ist. Dabei ist es interessant, dass eine Hemmung der Phospholipase $\mathrm{A}$ in Lysosomen der Lunge 
durch Ambroxol beobachtet wurde [26]. Die $\mathrm{IC}_{50}$ für diesen Effekt lag allerdings bei 200-500 $\mu \mathrm{M}$, eine Konzentration, die in vivo höchstens kurzfristig und lokalisiert erreicht wird.

In unserem Zellkulturmodell ist ein solcher Mechanismus möglicherweise aufgrund des Fehlens von ausreichend extrazellulärem, wieder aufgenommenen Surfactantmaterial nicht beobachtet worden. Der Unterschied im Vergleich zu den Untersuchungen von Disse et al. [2] könnte aber auch in der unterschiedlich langen Inkubation der isolierten Zellen begründet sein. Es wurde gezeigt, dass AT-II-Zellen auf normalem Zellkulturplastik nach 48 h nicht mehr die Phospholipidverteilung aufweisen, die in frisch isolierten Zellen vorliegt $[27,28]$. Nach 4 Tagen haben AT-II-Zellen auf normalem Zellkulturplastik auch morphologisch erhebliche Veränderungen durchgemacht und weisen Merkmale von AT-I-Zellen auf $[29,30]$. Alleine dieser Effekt könnte einen veränderten Einbau von Vorläufermolekülen erklären.

Tendenziell war eine Verminderung sowohl der stimulierten Sekretion als auch der unbeinflussten Synthese bei einer Konzentration von $10^{-5} \mathrm{M}$ Ambroxol im Vergleich $\mathrm{zu} 10^{-6} \mathrm{M}$, $10^{-7} \mathrm{M}$ und $10^{-8} \mathrm{M}$ Ambroxol zu erkennen. Diese Tendenz, die bei der Sekretion deutlicher war, zusammen mit der Beobachtung einer vermehrten LDH-Freisetzung bei noch höheren Konzentrationen $\left(10^{-4} \mathrm{M}, 10^{-3} \mathrm{M}\right)$ überrascht insofern, als beim Menschen im Lungengewebe nach Infusion von $1000 \mathrm{mg}$ Ambroxol über $3 \mathrm{~h}$ in den darauf folgenden $12 \mathrm{~h}$ ein mittlerer Spiegel von $27 \mu \mathrm{g}$ Ambroxol/g Lungengewebe berichtet wurde (also etwa $5^{*} 10^{-5} \mathrm{M}$ ) [31]. Somit erreichen die Gewebespiegel zumindest im Falle der Infusionstherapie selbst im Mittel einen Konzentrationsbereich, der in der Zellkultur zu einer tendenziellen Verminderung der Surfactantsekretion und bei längerer Inkubation auch zu einer vermehrten $\mathrm{LDH}$-Freisetzung als Zeichen gestörter zellulärer Integrität führt. Maximale Spiegel berichteten die Autoren im Bereich von $10^{-4} \mathrm{M}$ (bzw. $42 \mathrm{ng} / \mathrm{g}$ Lungengewebe). In der genannten Studie kam es dabei zu keinen erkennbaren Nebenwirkungen.

Ein statistisch signifikanter Unterschied im Sinne einer Verminderung der Surfactantsekretion/synthese bei einer Ambroxoldosis von $10^{-5} \mathrm{M}$ oder mehr hätte sich in der vorliegenden Untersuchung nach einer Abschätzung der geforderten Differenz und der beobachteten Standardabweichungen erst bei einem n von 30-50 ergeben können, und war daher nicht durchführbar.

Unsere Ergebnisse deuten also daraufhin, dass Ambroxol keine direkte Wirkung auf isolierte AT-II-Zellen besitzt, die zu einer signifikanten Steigerung der Surfactantsynthese und/ oder -sekretion führt. Der vermehrte Einbau von markierten Surfactantvorstufen in Surfactantphospholipide in vivo mag durch einen verminderten Abbau (und damit vermehrten Wiedereinbau von vorhandenem Surfactant) oder durch eine indirekte Stimulation durch Sekretionsprodukte (z.B. anderer Zellen der Lunge) zustande kommen. Es ist auch denkbar, dass Ambroxol in manchen Modellen durch seine Tumornekrosefaktor-alpha (TNF- $\alpha$ ) inhibierende Wirkung [32] wirksam wird, da TNF- $\alpha$ seinerseits in der Lage ist, die Surfactantsynthese zu hemmen [33]. Unabhängig davon besitzt Ambroxol wie bereits erwähnt, antioxidative und antiinflammatorische Effekte [20].
Dem Kliniker bleibt damit bedauerlicherweise weiterhin keine sichere Möglichkeit, das Surfactantsystem pharmakologisch spezifisch zu stimulieren, wenn man von der Stimulation der AT-II-Zellen im Rahmen einer $\beta_{2}$-adrenergen Stimulation und von der zumindest in vitro bestehenden Möglichkeit der Surfactantstimulation durch Lungendehnung [33] einmal absieht. Gerade eine derartige therapeutische Option aber wäre in vielen Situationen wünschenswert und es bleibt zu hoffen, dass die Beeinflussung der Surfactantsynthese, vielleicht aber noch wichtiger der Surfactantsekretion ein Ziel pharmazeutischer Entwicklungsbemühungen wird. In dieser Situation stellt auch die exogene Surfactantapplikation außer für das Atemnotsyndrom des unreifen Neugeborenen keine gute Alternative dar, denn sie kann aus Gründen der Invasivität und der hohen Kosten nur in extremen Situationen und nur über einen sehr begrenzten Zeitraum durchgeführt werden.

\section{Literatur}

${ }^{1}$ Van Golde LMG, Post M, de Vries ACJ, Batenburg JJ. Type II cells isolated from adult and fetal rat lung as a model for studies on the formation of pulmonary surfactant. In: Cosmi EV, Scarpelli EM (Hrsg). Pulmonary surfactant system. Elsevier: Amsterdam, 1983: $57-72$

${ }^{2}$ Disse BG, Mason RJ, Voelker DR. Influence of ambroxol on surfactant lipids of rat alveolar type II cells in primary culture. Eur J Respir Dis 1987; 71 Suppl 153: 288-289

${ }^{3}$ Dobbs LG. Isolation and culture of alveolar type II cells. Lung Cell Mol Physiol 1990; 2: L134 - L147

${ }^{4}$ Mason RJ. Surfactant secretion. In: Robertson B, Van Golde LMG and Batenburg JJ (Hrsg.). Pulmonary surfactant: from molecular biology to clinical practice. Elsevier: Amsterdam, 1992: 295312

${ }^{5}$ Dobbs LG, Gonzalez RF, Williams MC. An improved method for isolating type II cells in high yield and purity. Am Rev Respir Dis 1986; 134: 141 - 145

${ }^{6}$ Wirtz H, Schmidt M. Acute influence of cigarette smoke on secretion of pulmonary surfactant in rat alveolar type II cells in culture. Eur Respir J 1996; 9: 24-32

${ }^{7}$ Folch J, Lees M, Stanley GHS. A simple method for the isolation and purification of total lipides from animal tissues. J Biol Chem 1957; 226: $497-509$

${ }^{8}$ Fanestil DD, Barrows CH. Aging in the rotifer. J Gerontol 1965; 20: $462-469$

${ }^{9}$ Cerutti P, Kapanci Y. Effects of metabolite VIII of Bromexine (Na872) on type II epithelium of the lung. Respiration 1979; 37: $241-251$

${ }^{10}$ Stanley MW, Henry-Stanley MJ, Gajl-Peczalska J, Bitterman P. Hyperplasia of type II pneumocytes in acute lung injury. Am J Clin Pathol 1992; 97: 669-677

${ }^{11}$ Zavattini G, Leproux B, Daniotti S. Ambroxol. In: Braga PC, Allegra L (Hrsg.). Drugs in bronchial mucology. Raven Press Ltd: New York, 1989: 262 - 291

${ }^{12}$ Felix K, Pairet M, Zimmermann R. The antioxidative activity of the mucoregulatory agents: ambroxol, bromhexine and $\mathrm{N}$ acetyl-L-cysteine. A pulse radiolysis study. Life Sci 1996; 59: $1141-1147$

${ }^{13}$ Gillissen A, Bartling A, Schoen S, Schultze-Werninghaus WG. Antioxidant function of ambroxol in mononuclear and polymorphonuclear cells in vitro. Lung 1997; 175: 235-242

${ }^{14}$ Gillissen A, Jaworska M, Orth M, Coffiner M, Maes P, App EM, Cantin AM, Schultze-Werninghaus WG. Nacystelyn, a novel lysine salt of $\mathrm{N}$-acetylcysteine, to augment cellular antioxidant defence in vitro. Respir Med 1997; 91: 159-168 
${ }^{15}$ Gillissen A, Scharling B, Jaworska M, Bartling A, Rasche K, Schultze-Werninghaus WG. Oxidant scavenger function of ambroxol in vitro: a comparison with N-acetylsteine. Res Exp Med (Berl) 1997; 196: 389-398

${ }^{16}$ Nowak D, Antczak A, Krol M, Bialasiewicz P, Pietras T. Antioxidant properties of Ambroxol. Free Radic Biol Med 1994; 16: $517-522$

${ }^{17}$ Nowak D, Antczak A, Pietras T, Bialasiewicz P, Krol M. Protective effect of ambroxol against heat- and hydrogen peroxide-induced damage to lung lipids in mice. Eur Respir J 1994; 7: 1629-1634

18 Piotrowski WJ, Pietras T, Kurmanowska Z, Nowak D, Marczak J, Marks KJ, Mazerant P. Effect of paraquat intoxication and ambroxol treatment on hydrogen peroxide production and lipid peroxidation in selected organs of rat. J Appl Toxicol 1996; 16: $501-507$

${ }^{19}$ Suzuki M, Teramoto S, Matsuse T, Ohga E, Katayama H, Fukuchi Y, Ouchi Y. Inhibitory effect of ambroxol on superoxide anion production and generation by murine lung alveolar macrophages. J Asthma 1998; 35: $267-272$

${ }^{20}$ Winsel K. Antioxidative und entzündungshemmende Eigenschaften von Ambroxol. Pneumologie 1992; 46: 461 - 475

${ }^{21}$ Post M, Batenburg JJ, Schuurmanns EAJM, Oldenborg V, van der Molen AJ, van Golde LMG. The perfused rat lung as a model for studies on the formation of surfactant and the effect of ambroxol on this process. Lung 1983; 161: 349-359

${ }^{22}$ Kapanci Y, Elemer G. Ambroxol and surfactant secretion. Experimental studies on the incorporation of ${ }^{3} \mathrm{H}$-palmitate into pulmonary surfactant. In: Cosmi ES, Scarpelli EM (Hrsg.). Pulmonary surfactant system. Elsevier: Amsterdam, 1983: 263 - 272

${ }^{23}$ Lachmann B. The effect of ambroxol in newborn and adult animals with surfactant deficiency. In: Cosmi EV, Scarpelli EM (Hrsg.). Pulmonary surfactant system. Elsevier: Amsterdam, 1983: $237-248$

${ }^{24}$ von Wichert $\mathrm{P}$, Bavendamm $\mathrm{U}$, Teichmann $\mathrm{M}$ et al. Increased incorporation of fatty acids into phospholipids of lung and livers of rabbit under the influence of bromhexine and ambroxol. Arch Pharmacol 1977; 297: 269-273

25 Prevost MC, Soula G, Douste-Blazy L. Biochemical modifications of pulmonary surfactant after bromhexine derivative injection. Respiration 1979; 37: $215-219$

${ }^{26}$ Heath MF, Jacobson W. The inhibition of lysosomal phospholipase A from rabbit lung by ambroxol, and its consequences for pulmonary surfactant. Lung 1985; 163: $337-344$

${ }^{27}$ Dobbs LG, Mason RJ, Williams MC, Benson BJ, Sueishi K. Secretion of surfactant by primary cultures of alveolar type II cells isolated from rats. Biochim Biophys Acta 1982; 713: 118-127

${ }^{28}$ Mason RJ, Dobbs LG. Synthesis of phosphatidylcholine and phosphatidylglycerol by alveolar type II cells in primary culture. J Biol Chem 1980; 255: 5101 - 5107

${ }^{29}$ Dobbs LG, Williams MC, Brandt AE. Changes in biochemical pattern of lectin binding of alveolar type II cells with time in culture. Biochem Biophys Acta 1985; 846: 155-166

${ }^{30}$ Dobbs LG, Williams MC, Gonzalez R. Monoclonal antibodies specific to apical surfaces of rat alveolar type I cells bind to surfaces of cultured, but not freshly isolated, type II cells. Biochem Biophys Acta 1988; 970: 146-156

${ }^{31}$ Mezzetti M, Colombo L, Marini MG, Crusi V, Pierfederici P, Mussini E. A pharmacokinetic study on the pulmonary tropism of ambroxol in patients under thoracic surgery. J Emergency Surg Int Care 1990; 13: 179-185

32 Bianchi M, Mantovani A, Erroi A, Dinarello CA, Ghezzi P. Ambroxol inhibits interleukin I and tumor necrosis factor production in human mononuclear cells. Agents Actions 1990; 31: 275 - 279

${ }^{33}$ Balibrea-Cantero JL, Arias-Diaz J, Garcia C, Torres-Melero J, Simon C, Rodriguez JM, Vara E. Effect of pentoxifylline on the inhibition of surfactant synthesis induced by TNF- $\alpha$ in human type II pneumocytes. Am J Respir Crit Care Med 1994; 149: 699-706

34 Wirtz HRW, Dobbs LG. Calcium mobilization and exocytosis after one mechanical stretch of lung epithelial cells. Science 1990; 250: $1266-1269$

\section{Prof. Dr. H. Wirtz}

Medizinische Klinik I und Poliklinik

Universität Leipzig

Johannisallee 32

04103 Leipzig

E-mail: wirtzh@medizin.uni-leipzig.de 